\section{Haselnuss- plus Erdnussallergie: Eher Zufall oder liegen Kreuzreaktionen vor?}

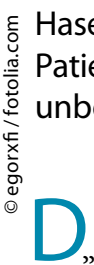
Haselnuss und Erdnuss sind botanisch zwar nicht miteinander verwandt, viele Patienten sind trotzdem gegen beide Pflanzen allergisch. Liegen doch bisher unbekannte Kreuzreaktivitäten vor oder ist die Koallergisierung nur Zufall?

ie Haselnuss (Corylus avellana) als „Frühblüher" ist botanisch verwandt mit Birke und Erle, nicht aber mit der Erdnuss (Arachis hypogaea), die zur Familie der Hülsenfrüchtler (Fabaceae/Leguminosae) gehört. Deshalb ist es unwahrscheinlich, dass die Proteine und damit die potenziellen Allergene beider Pflanzenarten molekularbiologisch übereinstimmen. Um das tatsächlich ausschließen zu können, wurden mögliche Kreuzreaktivitäten an einer retrospektiv gebildeten Kohorte von 161 Kindern und Erwachsenen mit einer Sensibilisierung gegen Haselnussallergene überprüft.

Per ImmunoCAP ermittelte man Sensibilisierungen gegen verschiedene Haselnuss- und Erdnussallergene. Bei 14 Patienten mit einer starken Reaktion gegen Erdnuss- oder Haselnuss-2S-Albumine (Cor a14, Ara h 2) und/oder 11S-Globuli- ne (nCor a 9, rAra h 3) wurden mögliche Kreuzreaktivitäten durch Inhibitionsversuche überprüft. Die klinische Diagnose erfolgte entweder per doppelblind und placebokontrolliert durchgeführter Provokation und/oder aufgrund von Symptomatik und Anamnese.

Von den Haselnuss-sensibilisierten Patienten waren $68 \%$ zusätzlich gegen Erdnussallergene sensibilisiert, $43 \%$ zeigten klinische Symptome einer Nahrungsmittelallergie gegen Erdnüsse. Diese Erdnussallergien waren aber nicht assoziiert mit dem Vorliegen oder der Schwere einer klinisch manifesten Haselnussallergie, Assoziationen bestanden lediglich mit einer IgE-Reaktivität gegen Erdnuss-Speicherproteine, insbesondere Ara h 2. Ara h 2 zeigte keine Kreuzreaktivitäten mit Cor a 14 . Bei zwei von 13 Patienten ließen sich allerdings deutliche Kreuzreaktivitäten zwi- schen den 11S-Globulinen nachweisen: Ara h 3 hemmte praktisch vollständig die IgE-Bindung von Cor a 9.

Fazit: Eine Erdnussallergie ist im Allgemeinen nicht auf Kreuzreaktionen gegen Haselnussallergene zurückzuführen. Eine zusätzliche Erdnussallergie bei Haselnuss-sensibilisierten Patienten scheint damit in der Regel zufällig zu sein. Sehr selten kann eine primäre Ara-h-3-Sensibilisierung mögliche Ursache einer $\mathrm{Ha}$ selnussallergie sein. Eine IgE-Reaktivität gegen Cor a 14 oder Ara h 2 sind aussagekräftige Marker für eine primäre Sensibilisierung gegen Haselnuss respektive Erdnuss.

Dr. Barbara Kreutzkamp

Masthoff LJ et al. Peanut allergy is common among hazelnut-sensitized subjects but is not primarily the result of IgE cross-reactivity. Allergy 2015; 70: 265-74

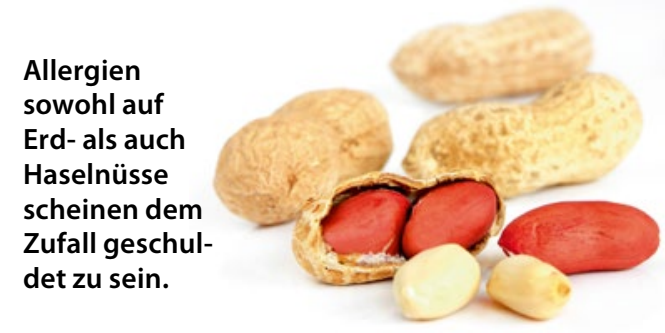

folgsraten nach dem Gerätetausch. Entscheidend war vielmehr, welcher AAI in der zweiten Phase verwendet wurde. Mit dem Auvi-Q ${ }^{\mathrm{TM}}$ konnten $93 \%$ der Eltern erfolgreich Adrenalin injizieren im Vergleich zu $49 \%$ mit allen anderen AAI. Besonders schlecht schnitten Eltern ab, die von einem AAI mit zwei Sicherheitskappen $\left(\right.$ Anapen $^{\circledast}$ ) auf einen mit einer Kappe (alle anderen) wechselten und umgekehrt. Die Autoren empfehlen Allergologen, diesen Umstand zu berücksichtigen, wenn ihre Patienten den AAI wechseln.

Fazit: Die Beschaffenheit eines AAI scheint entscheidend zu beeinflussen, ob das jeweilige Gerät korrekt angewendet wird. Audiogestützte AAI erzielen die besten Erfolgsraten. Sebastian Lux

Umasunthar et al. Patients' ability to treat anaphylaxis using adrenaline autoinjectors: a randomized controlled trial. Allergy 2015; 70: 855-63 